\section{Multiple malformation syndrome including cleft lip and palate and cardiac abnormalities due to an interstitial deletion of chromosome $12 \mathrm{q}$}

The proband, a male, was the second child of young, healthy parents. The older brother is healthy. The pregnancy progressed normally with spontaneous delivery at 39 weeks. Birth weight was $2030 \mathrm{~g}$, length $44 \mathrm{~cm}$, and head circumference $33 \mathrm{~cm}$. Craniofacial dysmorphism included hypertelorism, upward slanting palpebral fissures, broad nasal bridge, bilateral cleft lip and palate, macrostomia, retrognathia, and deep set, dorsally rotated auricles. There was overriding of the second and fifth fingers bilaterally, rocker bottom feet, and short big toes. Cardiac findings suggested an atrial septal defect and a superior ventricular septal defect. Recurrent vomiting and haematemesis was observed and the child's development was retarded. He died at 10 weeks and the parents refused necropsy.

Chromosomal analysis of lymphocyte cultures showed an interstitial deletion of the long arm of chromosome 12: $46, X Y, \operatorname{del}(12)(q 13 \cdot 3 \rightarrow q 21 \cdot 1)$. Fibroblast cultures obtained post mortem confirmed this finding. Parental karyotypes were normal.

The phenotype of the child was suggestive of trisomy 18 . The cleft lip and palate could be consistent with this diagnosis, as about $25 \%$ of patients with trisomy 18 exhibit

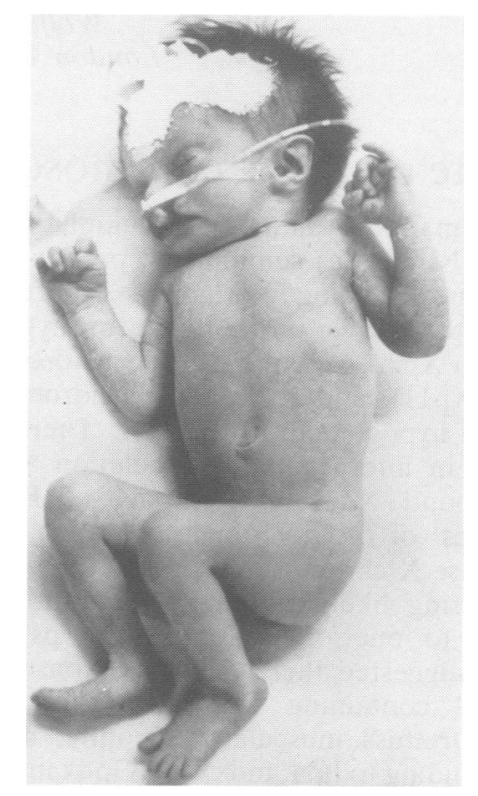

FIG 1 The proband with interstitial deletion $12 q$.

Received for publication 30 August 1985 .

Accepted for publication 9 September 1985

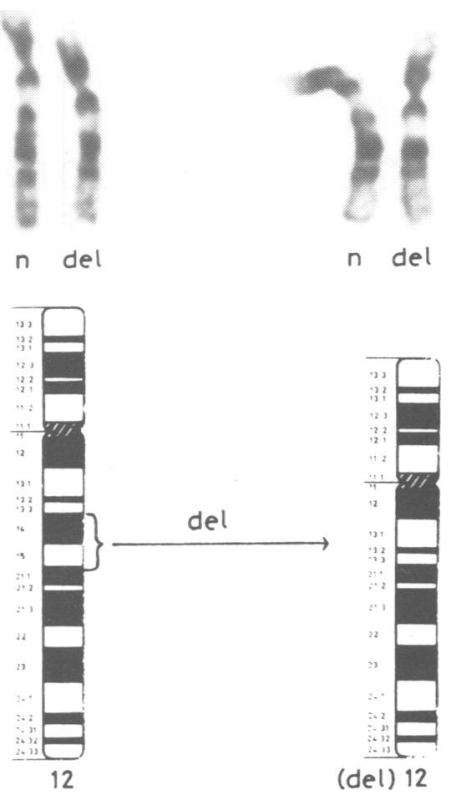

FIG 2 GTG banded chromosome 12 from two different cells and diagrammatic representation of the interstitial deletion ( $n=$ normal, del=deletion).

this feature. ${ }^{1}$ It appears that interstitial deletion of the long arm of chromosome 12 is able to mimic the phenotype of trisomy 18 to a considerable extent. To our knowledge, no similar case of interstitial deletion $12 \mathrm{q}$ has yet been reported, and recent text books do not provide any appropriate references. ${ }^{12}$ Therefore, it is not certain whether or not a constant karyotype/phenotype correlation exists. If this correlation does exist, then this type of interstitial deletion could be described as another example of 'pseudo-trisomy 18'. A further nosological aspect of this case is to add another example to an already lengthy list of syndromes which feature cleft lip and palate. ${ }^{2}$

\section{Peter Meinecke and Renate Meinecke Humangenetische Untersuchungsstelle, 2000 Hamburg 50, Federal Republic of Germany.}

\footnotetext{
References

1 Schinzel A. Catalogue of unbalanced chromosome aberrations in man. Berlin, New York: de Gruyter, 1984.

2 Borgaonkar DS. Chromosomal variation in man. A catalog of chromosomal variants and anomalies. 4th ed. New York: Alan R Liss, 1984.

${ }^{3}$ Cohen MM Jr. Syndromes with cleft lip and cleft palate. Cleft Palate J 1978;15:306-28.

Correspondence and requests for reprints to $\mathrm{Dr}$ Peter Meinecke, Humangenetische Untersuchungsstelle, Buelowstrasse 9, 2000 Hamburg 50, Federal Republic of Germany.
} 